\title{
High-Strength Undiffused Brushless (HSUB) Machine
}

\author{
John S. Hsu, Seong-Taek Lee, and Leon M. Tolbert \\ Oak Ridge National Laboratory \\ 2360 Cherahala Boulevard \\ Knoxville, Tennessee 37932, U.S.A.
}

\begin{abstract}
This paper introduces a new high-strength undiffused brushless machine that transfers the stationary excitation magnetomotive force to the rotor without any brushes. For a conventional permanent magnet (PM) machine, the air gap flux density cannot be enhanced effectively but can be weakened. In the new machine, both the stationary excitation coil and the PM in the rotor produce an enhanced air gap flux. The PM in the rotor prevents magnetic flux diffusion between the poles and guides the reluctance flux path. The pole flux density in the air gap can be much higher than what the $P M$ alone can produce. A high-strength machine is thus obtained. The air gap flux density can be weakened through the stationary excitation winding. This type of machine is particularly suitable for electric and hybrid-electric vehicle applications. Patents of this new technology are either granted or pending.
\end{abstract}

\section{INTRODUCTION}

The objective of this paper is to introduce a new "highstrength undiffused brushless (HSUB)" [1] type of interior permanent magnet (PM) electric machine for electric vehicle (EV) and hybrid electric vehicle (HEV) traction motors. The required torque/speed curve of a traction motor is shown in Fig. 1. A high torque is needed to start and accelerate the vehicle. After the base speed is reached, the required torque gradually diminishes along a constant power curve. A constant power speed ratio (CPSR) is the ratio of the highest possible speed delivering the base power to the base speed, which is the highest speed at which rated torque may be delivered.

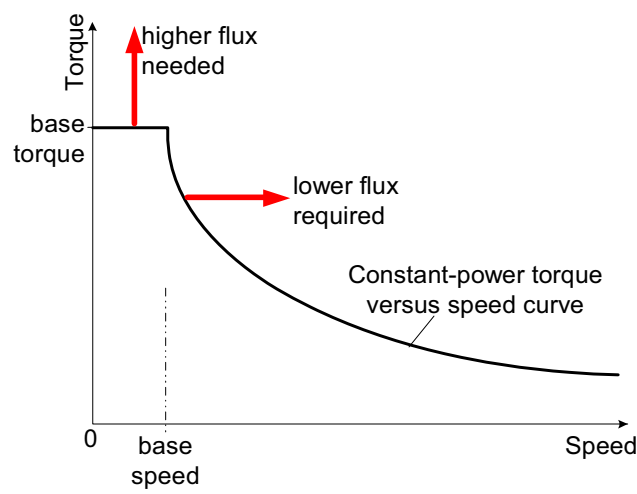

Fig. 1. A typical required torque/speed curve for electric vehicle motor drives.
Because for a given maximum current the motor torque is proportional to the pole flux, an elevated torque requirement during start-up and acceleration requires that motor pole flux be increased. Above base speed, the motor pole flux needs to be weakened for the needed current, which is limited by the voltage, to produce a high CPSR.

Among interior-PM reluctance motors, three different PM strength levels are shown in Fig. 2. They range from no PM to strong PM in grooves. The PM helps to produce higher torque as a result of higher pole flux density with a given stator current amplitude; on the other hand, a strong PM also is associated with higher core loss and significant back electromotive force at high speed.

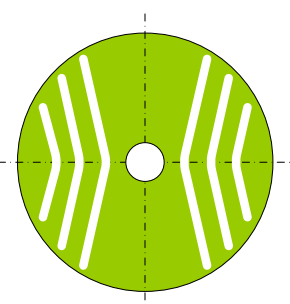

No PM in Grooves No PM torque Low reluctance torque Low iron loss at high speed Higher copper loss at low speed No back em

Lower voltage for high speed

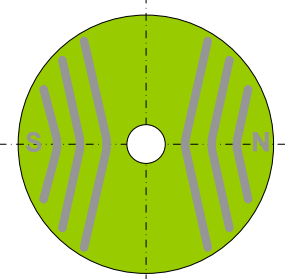

Weak PM in Grooves Weak PM torque Medium reluctance torque Medium iron loss at high speed Medium back emf
Medium voltage for high speed Medium copper loss at low speed

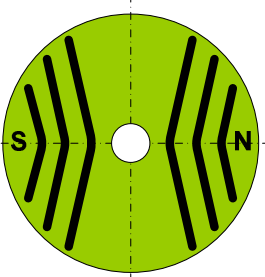

Strong PM in Grooves Strong PM torque Strong reluctance torque High iron loss at high speed Lower copper loss at low speed High back emf

High voltage required for high speed
Fig. 2. No-PM, weak-PM, and strong-PM reluctance rotors.

The HSUB machine discussed in this paper offers a controllable choice for the pole flux density. The rotor pole flux density can be significantly enhanced and weakened through the brushless field excitation coils.

Unlike the HSUB machine, many existing technologies [2-9] that deal with motor flux controls do not provide a significant field enhancement for the interior PM machine. In order to understand the physics for obtaining high strength under the field enhancement situation, the undiffused approach will be discussed first.

\footnotetext{
*The submitted manuscript has been authored by a contractor of the U. S. Government under contract No. DE-AC05-00OR22725. Accordingly, the U. S. Government retains a nonexclusive, royalty-free license to publish or reproduce the published form of this contribution, or allow others to do so, for U. S. Government purposes.

Research sponsored by the Oak Ridge National Laboratory managed by UT-Battelle, LLC for the U. S. Department of Energy under contract DE-AC05$00 \mathrm{OR} 22725$.
} 
When the magnetic flux carried by the ferrous material goes from the excitation coil to the rotor poles, flux leakage [9] becomes a major problem that needs to be solved. To distinguish the flux leakage between the rotor poles from the flux leakages elsewhere in an electric machine, the flux leakage between rotor poles is named "flux diffusion."

\section{SIMPLE TEST ON UNDIFFUSED CONCEPT}

Fig. 3a shows the excitation coil located adjacent to the main air gap, and Fig. $3 \mathrm{~b}$ shows the coil located away from the main air gap.

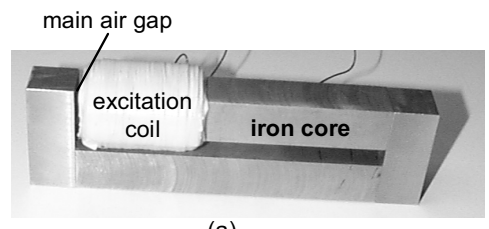

(a)

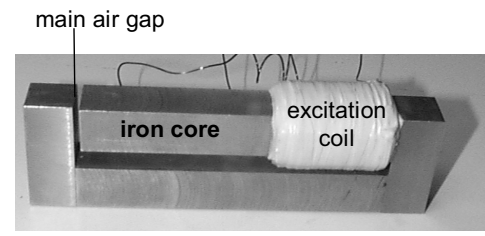

(b)

Fig. 3. Excitation coil locations: (a) close to air gap and (b) away from air gap.

The main air gap length in both cases of Fig. 3 is 2.8 $\mathrm{mm}(0.11$ inches). The relatively large gap accommodates a Hall-effect probe for measurement of the air gap flux density. In Fig. 4, the lower two traces show the main air gap flux densities versus the ampere-turns of the excitation coils for the two cases illustrated in Fig. 3. The upper linear trace gives the ideal flux density in the main air gap without flux diffusion and saturation.

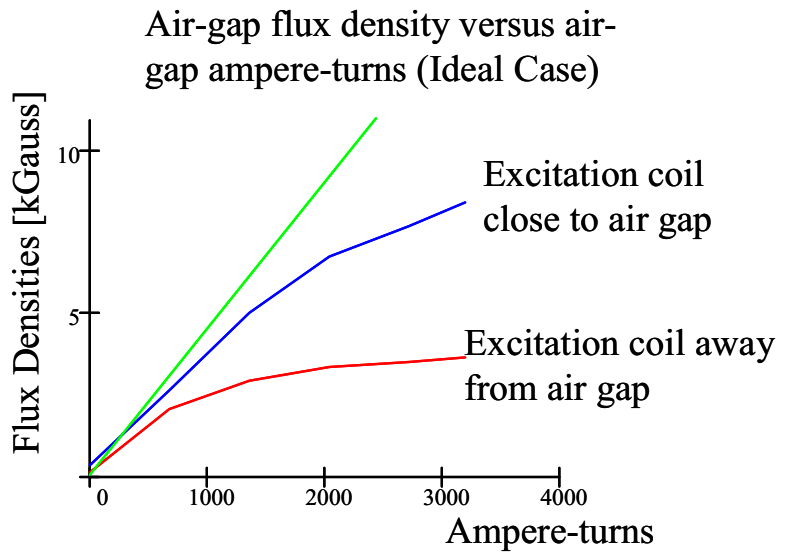

Fig. 4. Air gap flux densities versus ampere-turns produced by an excitation coil for two different coil locations.

The lowest trace indicates that for a given ampere-turn, the excitation coil located away from the air gap produces a low flux density in the air gap. This is because more flux diffusion happens along the iron core before the flux reaches the air gap. The middle trace shows a significant increase in the air gap flux density when the excitation coil is located close to the air gap. Comparing the middle trace and the top trace shows that when the excitation coil is located close to the air gap, most of the ampere-turns support the ampereturn drop of the air gap. The middle trace shows an increasing saturation effect when the flux density becomes higher. It should be noted that when the air gap is smaller, the flux densities of the three traces at a given ampere-turn would be higher than those shown in the figure. The traces would all be steeper.

\section{A. Tests of Undiffused Arrangements of a Rotor}

The proof-of-concept test in this section shows that when an excitation coil produces magnetic flux to increase the flux density of the main air gap, PMs can be used to discourage flux diffusion (or leakage).

Fig. 5 shows various undiffused arrangements for the original case of Fig. 3b, which has an excitation coil located away from the air gap. The undiffused arrangement is progressively increased from no undiffused arrangement in Fig. 5a to a highly undiffused arrangement in Fig. 5f, where most of the core of one side of the excitation coil is enveloped by PMs. Since this is a proof-of-concept test, available PMs were used without the luxury of complete excitation coil enclosure.

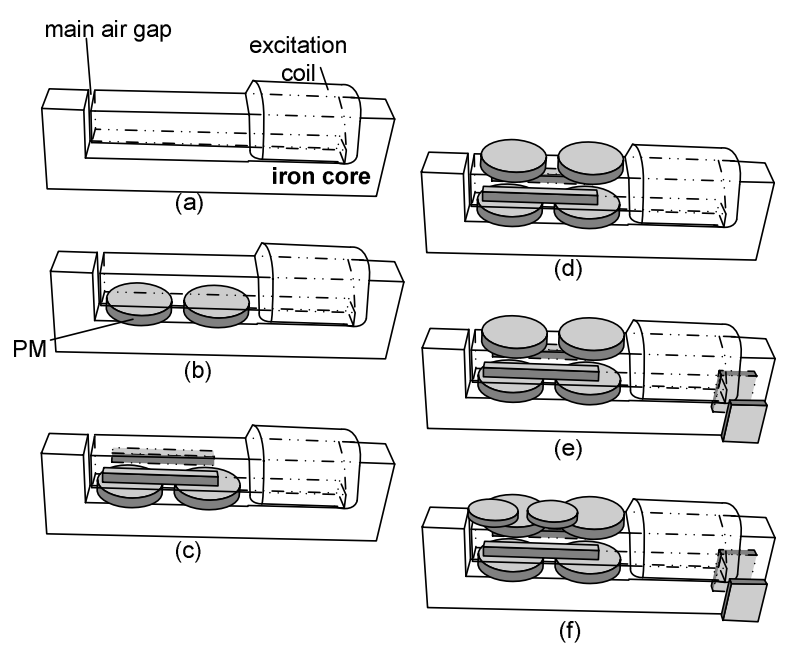

Fig. 5. Various degrees of undiffused arrangement.

Fig. 6 shows the measured main air gap flux density versus ampere-turns for the various degrees of undiffused arrangement shown in Fig. 5. 


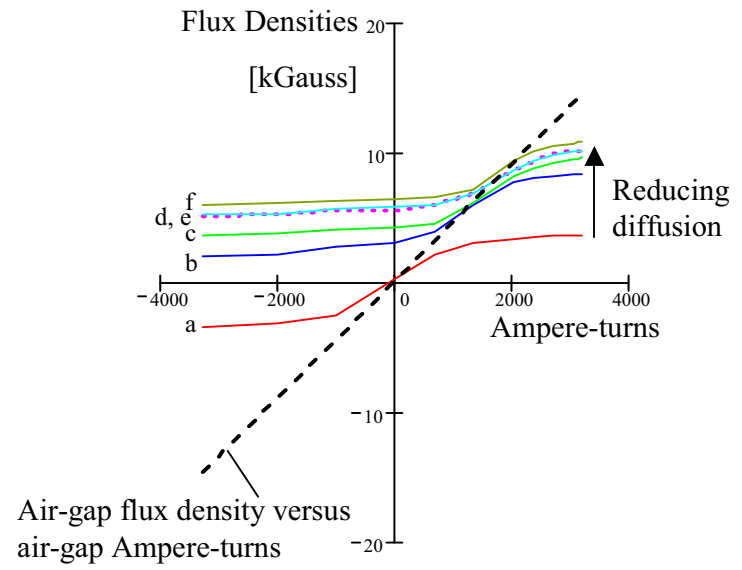

Fig. 6. Main air gap flux density versus ampere-turns for various degrees of undiffused arrangement.

The traces for arrangements $d$ and e in Fig. 6 show little difference when PMs are added on the other side of a pole, as shown in Figs. 5d and 5e. A good undiffused arrangement wrapped around a pole (i.e., one side of the excitation coil) is most effective.

For a given ampere-turn of the excitation coil, the main air gap flux density will go up when the gap is smaller. This is because of a lower ampere-turn drop across the main air gap and because of a lower counter-magnetomotive force acting on the PMs. The traces would tilt more toward the vertical direction.

\section{B. Excitation Air Gap and Main Air Gap}

The objective for brushless flux transfer to a rotor is to have a stationary dc-excitation coil that is not a part of the rotor. An additional air gap for the excitation coil is shown in Fig. 7. With reference to Fig. 5, only a portion of the undiffused PMs is shown in Figs. $7 \mathrm{~b}$ and $7 \mathrm{c}$.

Fig. 7a shows the magnetic path components marked with "Fe," the main air gaps on the left-hand side, and the excitation coil with its air gaps on the right-hand side. The two center $\mathrm{Fe}$ bars represent the rotor. When the current flows in the excitation coil, magnetic fluxes are produced. The main air gap flux is not the total flux produced by the coil. A significant portion of the flux is shown as the diffused flux. Fig. $7 \mathrm{~b}$ shows that to enhance the main air gap flux, a PM is placed between the upper and lower ferrous rotor pieces or around a pole, as shown in Fig. 5. The PM in the rotor produces flux in the main air gap and also prevents magnetic flux diffusion between the poles. Thus it enhances the usable main air gap flux density. Fig. $7 \mathrm{c}$ shows that reversing the current direction in the excitation coil can reduce the main air gap flux. This provides a simple field-weakening feature in the main air gap of this new machine.

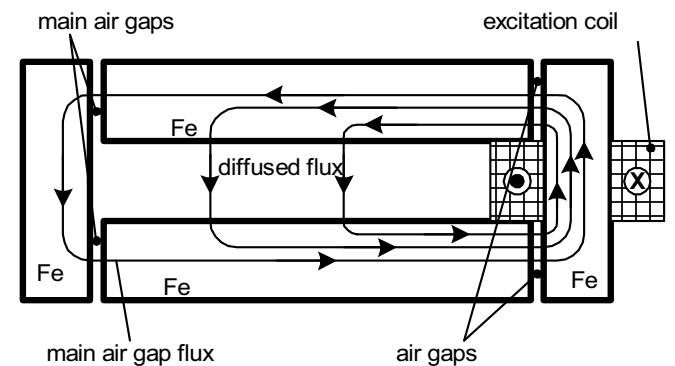

(a). Main air gap flux and diffused flux

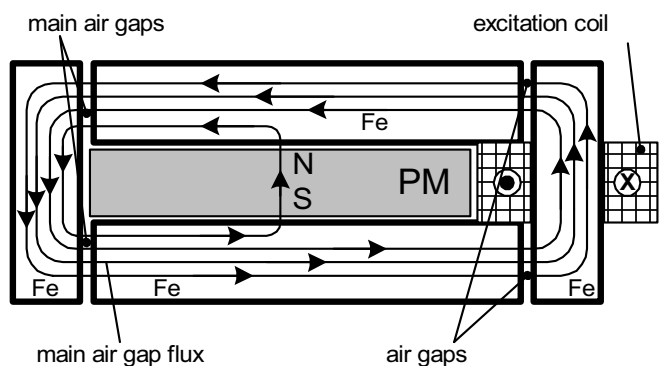

(b). Enhanced main air gap flux due to undiffused blocking provided by permanent magnet

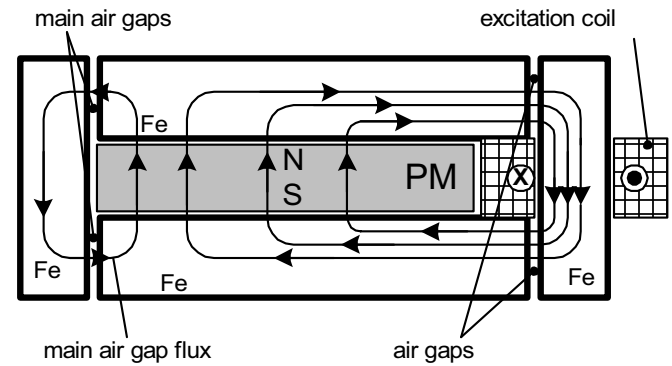

(c). Weakened main air gap flux due to reversed excitation

Fig. 7. Air gaps for the excitation coil: (a) main air gap flux and diffused flux without PM in rotor, (b) enhanced main air gap flux, (c) weakened main air gap flux.

\section{PROTOTYPE MACHINE}

The HSUB machine can be built as an axial-gap or a radial-gap machine. Fig. 8 shows the cut view of an axialgap HSUB prototype machine. It is brushless and consists of an armature, a rotor, and a dc-excitation stator. These three components are separated by air gaps. The armature has a set of polyphase windings and a magnetic core. When phase currents energize the polyphase windings, they produce a rotating magnetic wave in the main air gap. The rotor has two sides. One side faces the armature; the other side faces the dc-excitation stator. Rotor torque on the dcexcitation side, which is the derivative of the flux linkage of the dc-excitation coil with respect to the rotor angular displacement, is zero because of the unchanging dc flux. 




Fig. 8. Cut view of an HSUB machine.

\section{A. Axial-Gap Machine}

The outer and inner rotor rings are shown in Fig. 9. These two rings are brazed together to form the rotor. The PM material wrapped around the steel poles is injected into the space between the rotor outer and inner rings. The PM material acting as an undiffused flux barrier also produces the north and south poles on the side that faces the armature. Subsequently, the main air gap between the armature and the rotor sees the rotor flux interacting with the armature flux. This air gap flux can be either enhanced or weakened by the dc-excitation stator that faces the other side of the rotor.
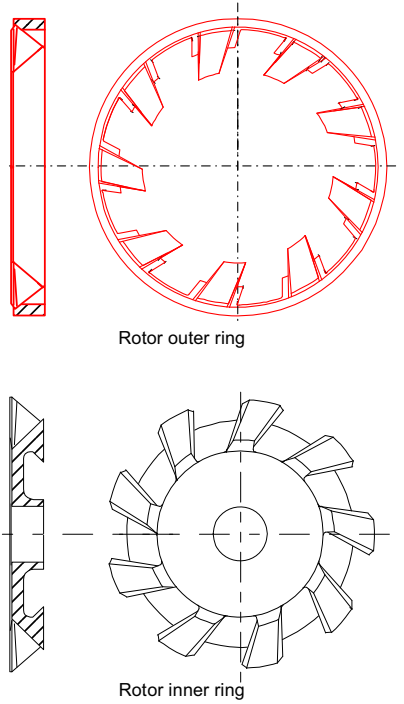

Fig. 9. An example of rotor outer and inner rings.
During field enhancement, the PM material in the rotor prevents diffusion of flux between the rotor magnetic poles, thereby guiding more flux to the main air gap to interact with the armature flux.

During field weakening, a great portion of the main air gap flux is diverted from the air gap by controlling the dc current in the dc-excitation stator. The core loss can be reduced by a lower flux density in the main air gap between the armature and the rotor.

A rotor end-view of the prototype machine is shown in Fig. 10, and the opposite end-view is shown in Fig. 11.

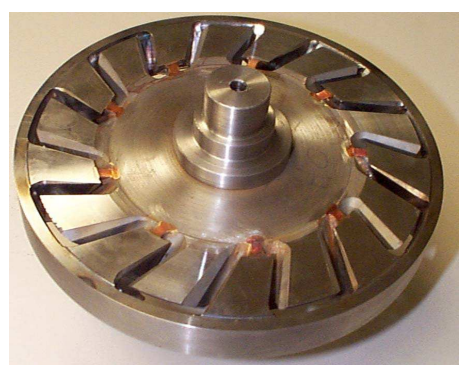

Fig. 10. Rotor end-view with poles of an HSUB machine before injection of PM material.

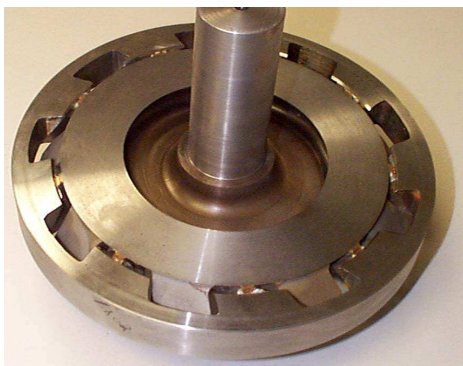

Fig. 11. Rotor opposite end for constant flux transfer of an HSUB machine before injection of PM material.

\section{B. Injected PM}

Injected PM material is used to fill the gaps between the rotor outer ring and inner ring of the prototype machine. A high residual flux density and a strong coercive force of the PM may improve the field-enhancement performance of the HSUB machine.

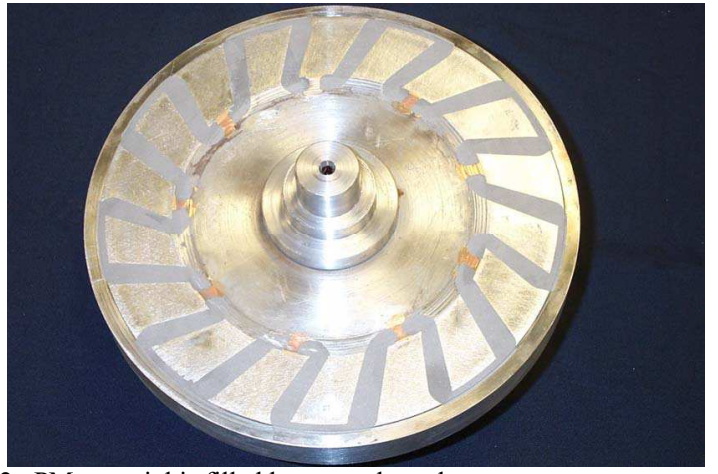

Fig. 12. PM material is filled between the poles.

Figs. 12 and 13 show where the PM material is placed to block the flux diffusion between poles. 


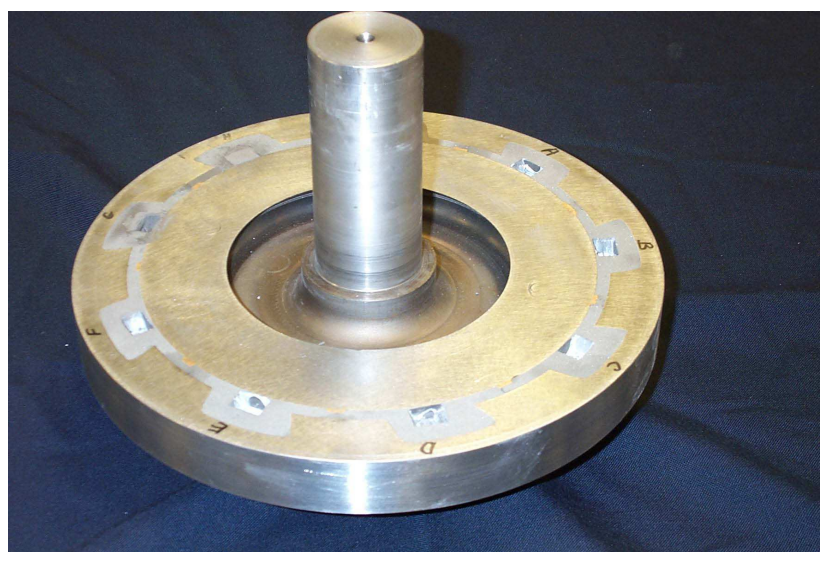

Fig. 13. PM material is filled between the poles on the side facing the field excitation.

\section{Test Setup and Test Results}

Fig. 14 shows the test setup of an HSUB prototype motor that has the rotor shown in Fig. 12. A torque gauge is attached between the HSUB machine shaft and the dynamometer shaft. Additionally, an optional rotor position encoder is mounted at the non-driving end of the HSUB machine shaft for running the machine as a brushless $\mathrm{dc}$ motor.



Fig. 14. Test setup of an HSUB prototype motor.

Fig. 15 shows a significant advantage of the HSUB motor. Based on the results of the locked-rotor-torque test, there is a torque increase of more than $50 \%$ with the PM of the rotor magnetized compared with the torque before it was magnetized. The PM material used in the prototype is Magnequench $\AA$ MQP-S spherical powder with $\mathrm{Br}=7.2 \mathrm{kG}$, $\mathrm{Hc}=8.3 \mathrm{kOe}$, and peak normal energy product $=9.5$ MGOe. The performance of the machine improves further if higher-quality PM material is used. A stronger coercive force would prevent the PM from being demagnetized by any abnormally high opposite fields or high armature currents.

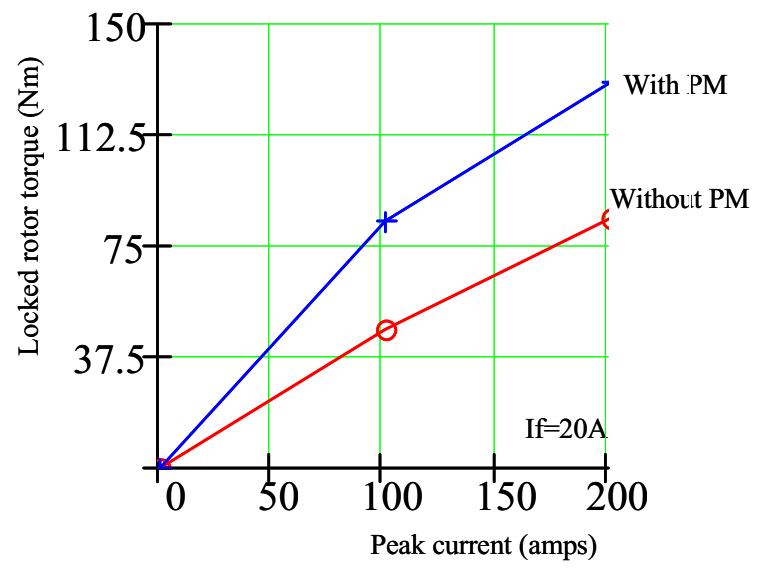

Fig. 15. Locked-rotor torques of HSUB prototype motor.

Fig. 16 shows the back-emf tested at $1000 \mathrm{rpm}$ under various excitation currents, If.

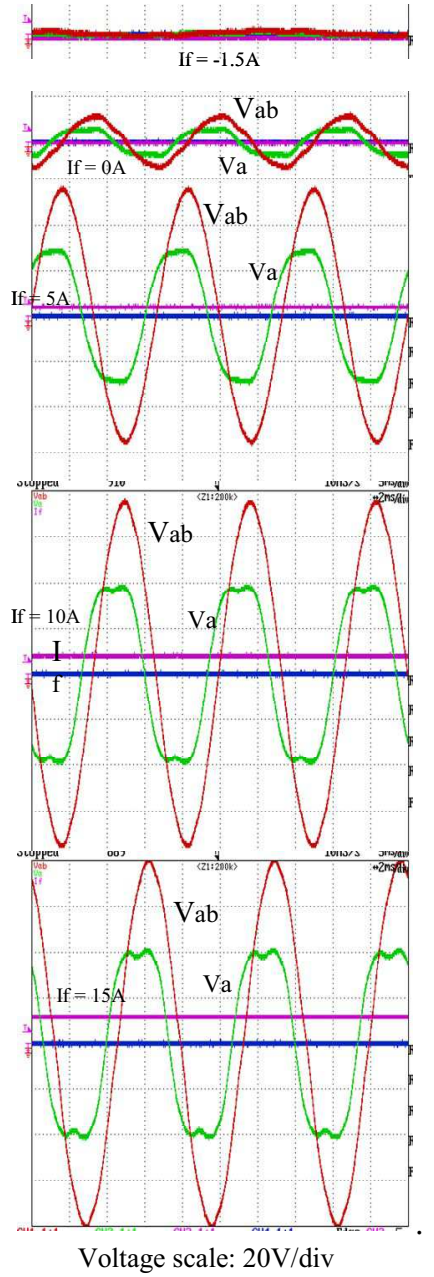

Fig. 16. Back-emfs tested at $1000 \mathrm{rpm}$.

The field weakening capability of the HSUB motor can be seen from the back-emf values of the motor driven as a generator. The back-emf can be controlled from zero to a 
high value. The zero back-emf is achieved by having a small negative excitation current. This controllable backemf ensures the capability for a wide speed ratio at the constant power region so that the HSUB motor can be used as an $\mathrm{EV}$ or HEV traction motor.

The phase back-emf, $V a$, induced from a full pitch winding with one coil per pole per phase, would naturally be a trapezoidal waveform. However, the line back-emf is close to a sinusoidal waveform.

Fig. 17 shows the combined mechanical and core losses of the prototype motor. These losses are low at low speed as a result of the lower frequency, even at high excitation for strong flux density. When the speed goes up, the frequency increases, and the mechanical and core losses are expected to go up. Fortunately, at high speed, the motor would be operating at the field-weakening region with lower flux density. The overall mechanical and core losses should be reasonably low in the entire operating region of the motor. Losses of few hundred watts are reasonable for a motor with a rating of a few tens of kilowatts.

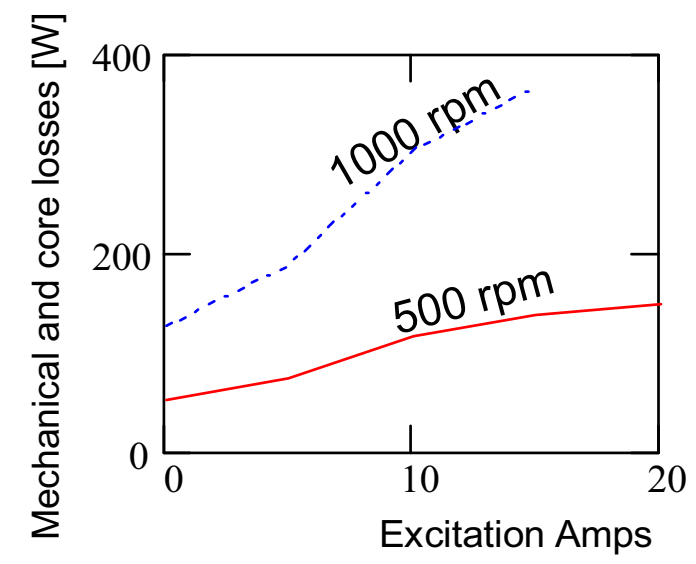

Fig. 17. Tested mechanical and core losses.

The HSUB machine in a motor mode can be operated as a synchronous motor without an encoder, or as a brushless de motor with a rotor position encoder. Fig. 18 shows the current and voltage traces of the prototype motor operating in the brushless de motor mode.

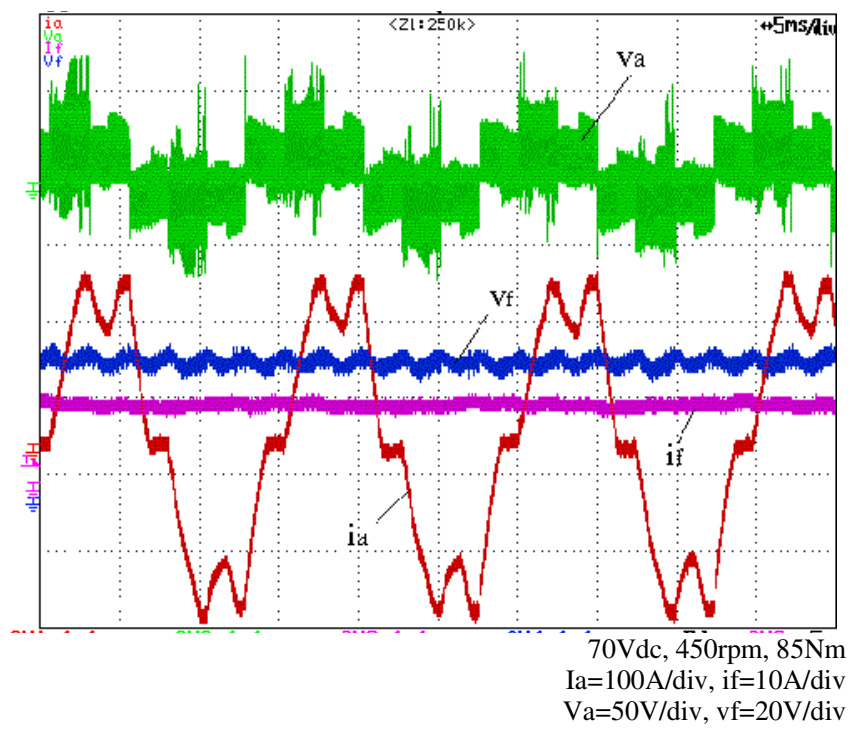

Fig. 18. Brushless dc-motor mode test at $450 \mathrm{rpm}$ loaded with $85 \mathrm{~N}-\mathrm{m}$ shaft torque.

\section{AdDitional Reluctance Torque}

Fig. 19 is a phasor diagram of the phase variables of a PM reluctance machine. The symbol $V$ is phase voltage; $I$, phase current; $E$, phase back-emf; suffix $d$, direct axis; $q$, quadrature axis; and $\delta$, load angle. The assumptions of sinusoidal time and space variables are used in the phasor diagram. The 3-phase power, $P$, going into the motor is derived through the products of the voltage projections and the currents.

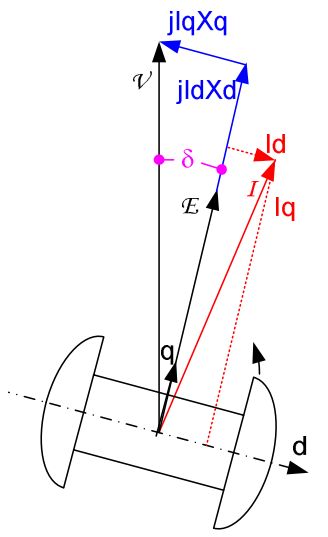

Fig. 19. Phasor diagram and symbol definitions of a PM reluctance motor.

$$
P=3\left(V \cos \delta I_{q}-\mathrm{V} \sin \delta I_{d}\right) .
$$

The $V \cos \delta$ and the $V \sin \delta$ terms of Eq. (1) can be rewritten according to Fig. 19. This gives

$$
P=3\left[\left(E+I_{d} X_{d}\right) I_{q}-I_{q} X_{q} I_{d}\right] .
$$


Substituting $I_{d}=(V \cos \delta-\mathrm{E}) / X_{d}$, and

$$
\begin{gathered}
I_{q}=(V \sin \delta) / X_{q} \text { into }(2) \text { gives } \\
P=3\left[V E \sin \delta / X_{d}+0.5 V^{2} \sin 2 \delta\left(1 / X_{q}-1 / X_{d}\right)\right] .
\end{gathered}
$$

The first term inside the brackets of (3) represents the synchronous power corresponding to the PMs that is proportional to $\sin \delta$. The second term is a typical reluctance power that varies according to $\sin 2 \delta$ and results from the difference of the reciprocals of the quadrature-axis and direct-axis reactances.

The torque capability of the motor measured at zero speed shown in Fig. 20 is relatively simple to obtain. This is accomplished by separating the tested torque into its fundamental and second harmonic components. The fundamental component will indicate the PM torque component, and the second-order harmonic will represent the additional reluctance torque produced by the reluctance difference.

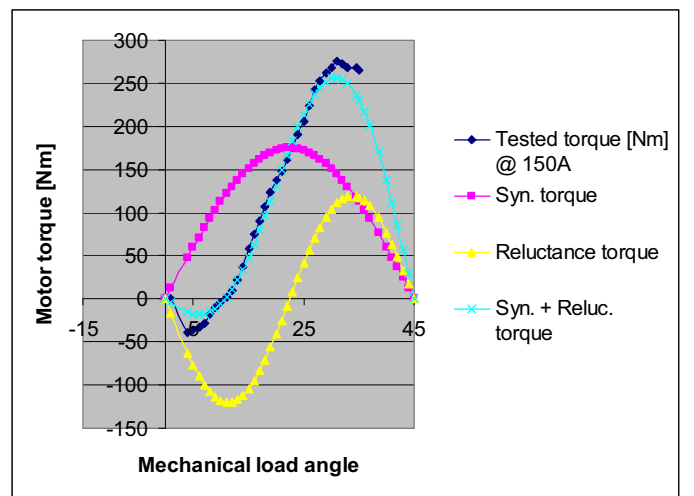

Fig. 20. Reluctance and PM torque components of a conventional interior $\mathrm{PM}$ reluctance motor.

\section{RADIAL-GAP HSUB MACHINE WITH RELUCTANCE TORQUE}

Fig. 21 shows a radial-gap HSUB machine with the capability to produce both $\mathrm{PM}$ and reluctance torque components. For the simulation, the core length of the stator is $2.5 \mathrm{in}$., the rotor diameter is $6.3 \mathrm{in}$., and the stator core outer diameter is 10.6in. The PM properties used in the simulation are $\mathrm{Br}=4 \mathrm{kG}$, and $\mathrm{Hc}=16 \mathrm{kOe}$. The low $\mathrm{Br}$ value indicates a relatively low-cost permanent magnet.

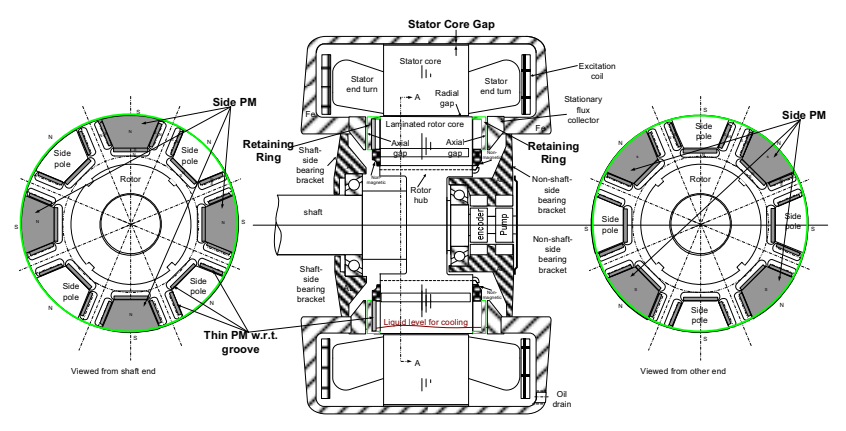

Fig. 21. A radial gap HSUB machine with reluctance torque component.

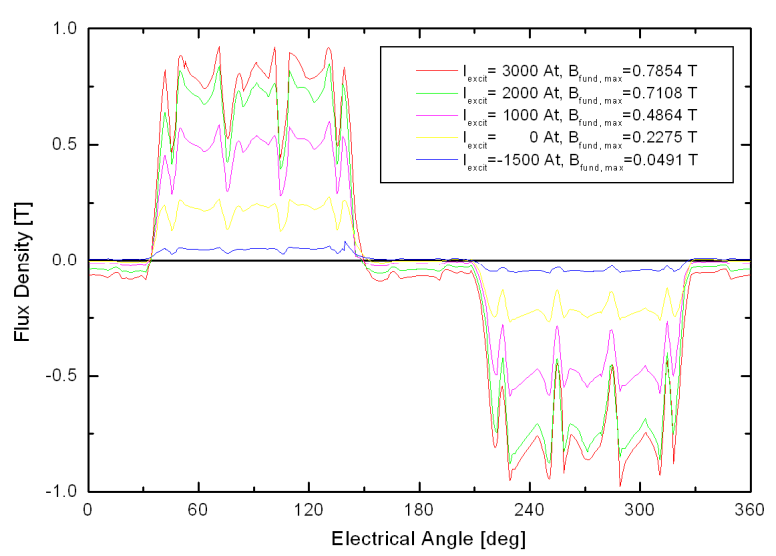

Fig. 22. Simulation results of air-gap flux density for different excitation values.

Fig. 22 shows that under the no-load condition, the airgap flux density can be changed significantly by changing the excitation ampere-turns. This indicates the field weakening and enhancement capabilities of the HSUB machine. The corresponding flux density vector distribution with 3000 ampere-turns field excitation is shown in Fig. 23.

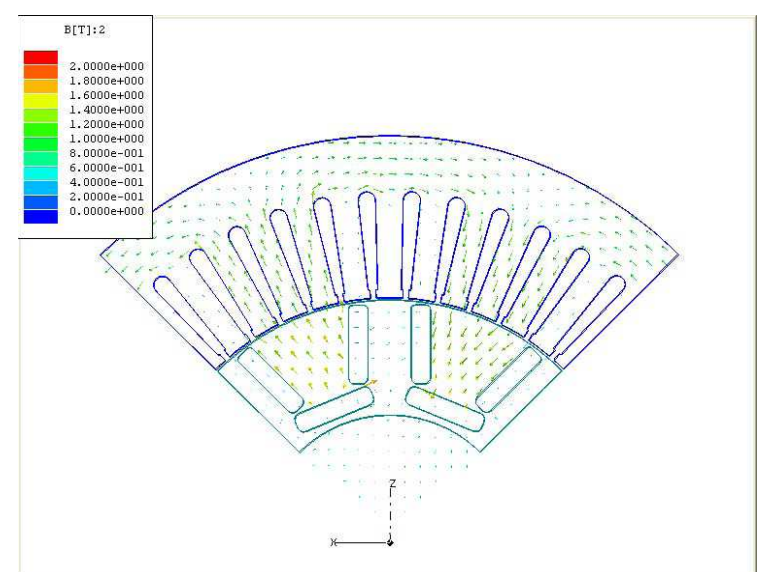

Fig. 23. No-load flux density vector distribution with 3000 ampere-turns field excitation.

Fig. 24 shows the flux density vector distribution at maximum torque position with stator current magnitude of $200 \mathrm{~A}$ and field excitation of 3000 ampere-turns. 


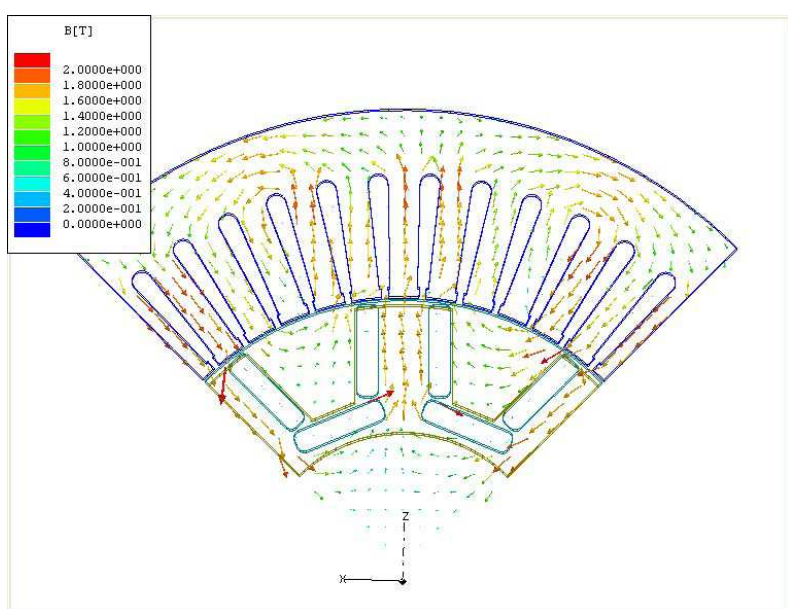

Fig. 24. Flux density vector distributions at maximum torque position (stator current amplitude $=200 \mathrm{~A}$ and field excitation $=3000 \mathrm{At}$ ).

Fig. 25 shows the comparison of output torque versus various excitation ampere-turn values. A torque increase over $60 \%$ can be achieved by flux enhancement.

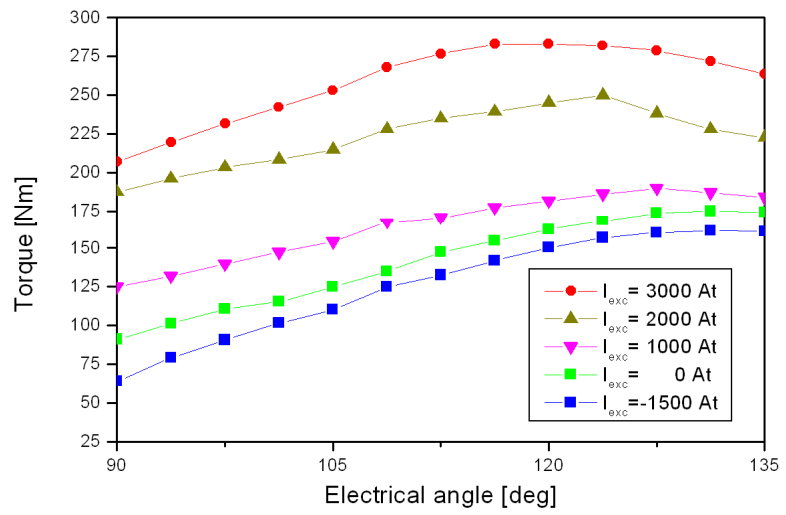

Fig. 25. Comparison of output torque under various excitation ampere-turn values.

\section{CONCLUSIONS}

- An HSUB machine is introduced. It can be built as an axial-gap or a radial-gap machine.

- The dc flux produced by an excitation coil is delivered to the rotor through an undiffused magnetic path without brushes.

- A prototype motor was built to examine the concept of its flux enhancement and weakening properties.

- Tests confirmed that the PM reluctance motor with an excitation coil can significantly enhance the air gap flux density; consequently, a high air gap torque can be obtained.

- The undiffused arrangement provided by PMs guides the flux to the main air gap facing the armature.
- A significant torque difference was measured between rotors with and without PMs for the undiffused arrangements.

- Controlling the current of the excitation coil can weaken the main air gap flux. This is proven by backemf tests; consequently, a high CPSR can be produced.

\section{ACKNOWLEDGMENT}

The authors appreciate the support from Ms. Susan Rogers, Manager, the Office of FreedomCAR and Vehicle Technology, Department of Energy. Encouragement from the Power Electronics and Electric Machinery Research Center headed by Mr. Donald Adams and managed by Dr. Mitchell Olszewski and Ms. Laura Marlino, and the building of experimental setups by Mr. Michael Jenkins, Sr., Mr. Curtis Ayers, and Mr. Chester Coomer are gratefully acknowledged.

\section{REFERENCES}

[1] J. S. Hsu, "High Strength Undiffused Brushless machines," U.S. Patent 6,573,634, June 3, 2003.

[2] H. Rosenberg, et al., "Permanent Magnet Excited Electric Machine," U.S. Patent No, 3,411,027, November 12, 1968.

[3] J. S. Hsu, "Hybrid-Secondary Uncluttered Induction (HSU-I) Machine," PES/IEEE Transactions on Energy Conversions, Paper No. PE-259EC, February 2001.

[4] J. S. Hsu, "Hybrid Secondary Uncluttered Induction Machine," U.S. Patent 6,310,417, October 30, 2001

[5] J. S. Hsu, "Simplified Hybrid-Secondary Uncluttered Machine and Method," U.S. Patent 6,891,301, May 10, 2005.

[6] J. S. Hsu, "Direct Control of Air Gap Flux in Permanent-Magnet Machines," pp. 361-365, PES/IEEE Transactions on Energy Conversions, 15(4) (December 2000)

[7] T. Mizuno, K. Nagayama, Tadashi Ashikaga, and Tadao Kobayashi, "Basic Principles and Characteristics of Hybrid Excitation Synchronous Machine," Electrical Engineering in Japan, 117 (5), (1996), translated from Denki Gakkai Ronbunshi, 115-D (11), pp. 1402-1411, (November 1995).

[8] J. S. Hsu, "Direct Control of Air gap Flux in Permanent-Magnet Machines," U.S. Patent 6,057,622, May 2, 2000.

[9] J. S. Hsu, "Flux Guides for Permanent-Magnet Machines," PES/IEEE Transactions on Energy Conversions, Paper No. PE-007EC, March 2001. 